Previous tumour predicts lung cancer risk

\section{Previous tumour as a predictor of risk of developing lung cancer}

\section{Eric M Toloza}

\section{The prognostic ability of a patient's history of a previous malignant tumour to predict the risk of developing lung cancer}

A n estimated 174470 new cases of lung cancer will be diagnosed and 162460 patients with lung cancer will die in the USA in 2006. ${ }^{1}$ Worldwide, a more staggering 1352132 new cases of lung cancer were diagnosed and 1178918 patients with lung cancer died in 2002. ${ }^{2} \mathrm{~A}$ low overall 5 year survival of $15-21 \%$ has been observed for all clinical stages combined due to findings of localised (or stage I or II) lung cancers in only $16-42 \%$ of all new cases of lung cancer. ${ }^{3}$

In order to improve survival among new cases of lung cancer, several efforts are being made to identify more patients with lung cancer while in the earlier stages (ie, small primary tumours with no mediastinal or distant metastasis) by both clinical and radiographic means. The use of chest radiograpphy with or without sputum cytology and the use of CT scans as screening tests for lung cancer have been evaluated. Five randomised controlled trials suggested that neither chest radiography nor sputum cytology prolonged life expectancies of individuals found to have lung cancer, despite detection of more cancers and more early stage cancers in the "screened" group than in the control group. However, this apparent lack of benefit from screening with chest radiography or sputum cytology has been criticised for the lack of a "no-screening" study arm in the randomised controlled trials or for being powered to detect a 50\% reduction in lung cancer mortality in the screened group compared with the control group rather than a smaller but still clinically meaningful $10 \%$ reduction in mortality.

Eight observational studies of lowresolution CT scanning with volunteer cohorts have shown that most lung cancers detected in these studies are stage I at the time of diagnosis. However, these studies have been criticised because detection of these early stage cancers may actually be the result of overdiagnosis of indolent non-aggressive malignancies in individuals in whom surgical treatment would not alter the natural history of the disease and in whom cancer treatment would not increase their life expectancies. ${ }^{4}$ The results of these studies have also been criticised because screened individuals as an aggregate, as well as society in general, may be harmed in excess of the benefit conferred to those detected to have lung cancer as a result of the high costs of screening and follow-up and the anxiety, morbidity and mortality associated with false-positive results. ${ }^{4}$ For example, in one series, while $35 \%$ of solitary pulmonary nodules (which were $<1 \mathrm{~cm}$ in diameter but which were suspicious enough to be an early stage lung cancer so as to warrant surgical biopsy) were indeed confirmed to be primary lung cancers and another 23\% were confirmed to be solitary metastases, $42 \%$ were actually benign lesions and thus resulted in patients having undergone unnecessary surgery. ${ }^{5}$ Thus, for individuals without either symptoms or a history of cancer, the use of sputum cytology, chest radiography or CT scanning as a screening test has not been recommended; however, individuals at risk of developing lung cancer were recommended to participate in screening trials. $^{6}$ In fact, the latest results of an international collaborative study shows that annual screening using low-dose spiral CT scanning of nearly 32000 asymptomatic persons at risk for lung cancer can detect clinical stage I lung cancers with estimated 10 year survival rates of $88 \%$ and as high as $92 \%$ if surgically resected within 1 month of diagnosis.

In order to improve the benefit and cost-effectiveness of these screening tests, clinical characteristics that give the clinician information on which to base a working diagnosis_-such as older age of the patient, associated symptoms (including respiratory tract symptoms such as cough, haemoptysis or chest pain or systemic symptoms of infection or inflammation), risk factors (including travel experience, tobacco history, asbestos exposure or history of prior cancer) and appearance of the lesion on the radiograph (including the length of time the lesion has been present) have been evaluated to determine which prognostic factors increase the probability that a solitary pulmonary nodule is malignant. ${ }^{58}$

Liu and colleagues showed that smoking is a risk factor for the development of multiple primary cancers, with upper aerodigestive tract tumours being the most frequent tumours accompanying lung cancer followed by colorectal and cervical cancer. ' Johnson and colleagues also showed that lung cancer survivors who continue to smoke cigarettes have an increased risk of developing a second lung cancer. ${ }^{10}$ Interestingly, a history of a prior tobacco-related malignancy was not an independent risk factor in the development of a subsequent malignancy, but all patients with lung cancer with a history or subsequent diagnosis of another primary malignancy were at a high risk for a third primary tumour (especially tobaccoassociated malignancies), with $74 \%$ of patients with lung cancer developing a new primary tumour within 2 years of their lung cancer diagnosis. ${ }^{11}$ Another study estimated that almost $82 \%$ of second primary tumours are diagnosed within 1 year following treatment for non-small cell lung cancer (NSCLC). ${ }^{12}$

Das and colleagues cited a number of previous studies showing that Hodgkin's disease survivors hade an increased risk of lung cancers and that radiation therapy, alkylating chemotherapy and smoking increased the risk of lung cancer in these patients; they then subsequently showed that patients with thoracic cancers after Hodgkin's disease presented at an advanced stage and had a poor survival. ${ }^{13}$ Askling and colleagues showed that a history of a squamous cell skin cancer increased the risk of death in patients who survived more than 1 year after the diagnosis of lung cancer. ${ }^{14}$ Similarly, a single institution review of patients with lung cancer who had one or more previous malignancies showed that a history of a prior tobacco-related malignancy was a risk factor for shortened survival. ${ }^{11}$ In a review of Medline articles published in English concerning lung cancers, second primary cancers, treatment of these cancers and patient survival, Johnson and colleagues found that the risk of developing a second lung cancer in patients with NSCLC who survived resection is approximately $1-2 \%$ per patient per year, and that the median survival from diagnosis of a second lung cancer in these patients is between 1 and 2 years with a 5 year survival of approximately $20 \% .^{10}$ In the same review, Johnson and colleagues found that the average risk of developing a second lung cancer in patients with small cell lung cancer (SCLC) who survived treatment is approximately $6 \%$ per patient per year, that the risk increases from 
approximately $2 \%$ to $>10 \%$ per patient per year 10 years after initial treatment, and that only $7 \%$ of patients with SCLC survive 2 years or more. ${ }^{10}$

In this issue of Thorax (see $p$ 386) the probability of death at 5 years in a patient with a completely resected pathological stage I NSCLC was shown to be increased by 1.5 times if that patient had had a previous tumour in a multi-institution study in which data from 2991 patients with lung cancer were collected prospectively from the time of diagnosis to the time of death, or at least to the time of last follow-up. ${ }^{15}$

Das and colleagues showed that CT screening for lung cancer may increase survival and quality-adjusted survival among Hodgkin's lymphoma survivors, with a benefit and incremental costeffectiveness ratio for smokers comparable to that of other recommended cancer screening strategies such as biennial mammography for breast cancer screening in 50-year-old women, annual Papanicolaou smears for cervical cancer screening in 20-year-old women, and colorectal cancer screening in 50-yearold men. ${ }^{16}$ Surprisingly, Duchateau and colleagues found that the 5 year survival rate was significantly better for patients with NSCLC who had more than two primary malignancies than for patients with NSCLC without any other primary malignancies and those with one other tumour in their history. They also found that the 5 year survival rate in patients with NSCLC with a second tumour in the follow-up period was better than in those without a second tumour. $^{12}$ Liu and colleagues also showed that the stage and median survival of patients with lung cancer who developed a second primary lung cancer were better than in patients who first had a non-lung cancer and who subsequently developed a subsequent primary lung cancer. ${ }^{9}$ Koppe and colleagues initially found similar results when their univariate analysis showed that a history of a previous malignancy was a favourable prognostic factor in the survival of patients with lung cancer. ${ }^{17}$ However, multivariate analysis showed tumour diameter, female gender and pathological TNM stage to be the major potential confounders. When adjustments were made for these three variables, the prognostic advantage of the previous malignancy disappeared, but nor was a prognostic disadvantage seen. ${ }^{17}$

Because of the increased risk of developing a second lung cancer, careful follow-up and intensive treatment is suggested for patients with lung cancer who are also deemed an important population for study of surveillance strategies and chemoprevention agents. ${ }^{90}$ Although there is no clear consensus for postoperative follow-up for patients with lung cancer or other malignancies, Brock and colleagues have recommend close (every 3-6 months) monitoring of the thorax with radiological imaging such as chest radiography or CT scanning, especially for the first 2 years when almost $75 \%$ of second primary tumours occurred. ${ }^{11}$ Koppe and colleagues also concluded that second primary NSCLC has a similar prognosis to first primary NSCLC and recommended that NSCLC diagnosed during the follow-up of a previous malignancy and deemed operable warrants the same diagnostic and therapeutic approach as NSCLC as first malignancy. ${ }^{17}$

\section{Thorax 2007:62:373-374.}

doi: $10.1136 /$ thx.2006.073478

Correspondence to: $\operatorname{Dr}$ Eric M Toloza,

Department of Surgery, Duke University Medical Center, Durham, North Carolina 27710, USA; toloz001@mc.duke.edu

Competing interests: None.

\section{REFERENCES}

1 Jemal A, Siegel R, Ward E, et al. Cancer statistics, 2006. CA: A Cancer Journal for Clinicians 2006;56:106-30.

2 Parkin DM, Bray F, Ferlay J, et al. Global cancer statistics, 2002. CA: A Cancer Journal for Clinicians 2005;55:74-108.

3 Mountain CF. Revisions in the International System for Staging Lung Cancer. Chest 1997;111:1710-7.

4 Bach PB, Kelley MJ, Tate RC, et al. Screening for lung cancer: a review of the current literature. Chest 2003;123:72-82S

5 Swanson SJ, Jaklitsch MT, Mentzer SJ, et al Management of the solitary pulmonary nodule: role of thoracoscopy in diagnosis and therapy. Chest $1999 ; 116: 523-4$ S.

6 Bach PB, Niewoehner DE, Black WC. Screening for lung cancer: the guidelines. Chest 2003;123:83-8S

7 Swensen SI, Silverstein MD, Ilstrup DM, et al. The probability of malignancy in solitary pulmonary nodules: application to small radiologically indeterminate nodules. Arch Intern Med 1997:57:849-55.

8 Tan BB, Flaherty KR, Kazerooni EA, et al. The solitary pulmonary nodule. Chest 2003;123:89-96S.

9 Liu Y-Y, Chen Y-M, Yen S-H, et al. Multiple primary malignancies involving lung cancer-clinical characteristics and prognosis. Lung Cancer 2002;35: 189-94.

10 Johnson BE. Second lung cancers in patients after treatment for an initial lung cancer. J Natl Cancer Inst 1998:90:1335-45.

11 Brock MV, Alberg AJ, Hooker CM, et al. Risk of subsequent primary neoplasms developing in lung cancer patients with prior malignancies. J Thorac Cardiovasc Surg 2004;127:1119-25.

12 Duchateau CSJ, Stokkel MPM. Second primary tumors involving non-small cell lung cancer: prevalence and its influence on survival. Chest 2005; 127:1152-8.

13 Das $\mathbf{P}, \mathrm{Ng} \mathrm{AK}$, Stevenson MA, et al. Clinical course of thoracic cancers in Hodgkin's disease survivors. Ann Oncol 2005; 16:793-7.

14 Askling J, Sørensen P, Ekbom A, et al. Is history of squamous-cell skin cancer a marker of poor prognosis in patients with cancer? Ann Intern Med 1999;131:655-9.

15 Lopez-Encuentra A, Gomez de la Camara A, RamiPorta R, et al. Previous tumour as a prognostic factor in stage I non-small cell lung cancer. Thorax 2007:62:386-90.

16 Das $\mathrm{P}, \mathrm{Ng}$ AK, Earle CC, et al. Computed tomography screening for lung cancer in Hodgkin's lymphoma survivors: decision analysis and costeffectiveness analysis. Ann Oncol 2006; 17:785-93

17 Koppe MJ, Zoetmulder FAN, van Zandwijk N, et al. The prognostic significance of a previous malignancy in operable non-small cell lung cancer Lung Cancer 2001;32:47-53.

\section{PDT in early central lung cancer}

\section{Lutz Freitag}

\section{Resources are needed to use the full potential of photodynamic therapy}

$\mathrm{n}$ this issue of Thorax, Moghissi et al ${ }^{1}$ report their experience of treating a selected group of patients with porphyrin-based photodynamic therapy (PDT) (see $p$ 391). These patients had localised early bronchogenic carcinomas without lymphadenopathy or distant metastases. They could not undergo surgery because of their overall clinical condition and half of them had been operated on before. Recognised as a world class thoracic surgeon, Mr Moghissi is certainly not questioning the value of surgery. Only after alternatives such as parenchymal-sparing bronchoplasty had been definitely excluded was PDT with curative intent offered. PDT was applied with a single laser light illumination using rigid bronchoscopy under general anaesthesia. Such a treatment usually takes less than 20 minutes, and at the Yorkshire Laser Centre it is performed as a day case procedure. 